\title{
Co-Occurrence of Apathy and Impulsivity in Progressive Supranuclear Palsy
}

Zi Q. Kok, BA (Hons), ${ }^{1}$ Alexander G. Murley, PhD, ${ }^{1,2}$ Timothy Rittman, PhD, ${ }^{1,2}$ (D) James Rowe, PhD, ${ }^{1,2,3}$ (D) and Luca Passamonti, PhD ${ }^{1,2,4, *}$ (D)

\begin{abstract}
Background: Apathy and impulsivity are common consequences of progressive supranuclear palsy (PSP) and can worsen its prognosis. They can co-exist in the same patients although their concomitant prevalence remains unclear. Their relationship to emotional lability is unknown.

Objectives: To estimate the co-occurrence of apathy and impulsivity and their relationship to emotional lability in PSP. To characterize the demographic, clinical, and cognitive features of PSP patients with apathy and impulsivity.

Methods: In a retrospective study of a long-term clinical cohort, we assessed the prevalence of apathy, impulsivity, and emotional lability from clinical interviews, medical records, and contemporary carer questionnaires. One hundred fifty-four patients with a diagnosis of probable or possible PSP (according to the 2017 Movement Disorder Society criteria) were identified. Sixty-four of these patients had neuropathological confirmation of PSP. PSP patients with both apathy and impulsivity were compared in terms of demographic, clinical, and cognitive characteristics to PSP patients with either one or neither of these neuropsychiatric features.

Results: Apathy and impulsivity co-existed in two-thirds of people with PSP. A fifth displayed emotional lability in addition to apathy and impulsivity. Apathy and impulsivity were more commonly co-expressed than by chance. There was no single demographic, clinical or cognitive feature that distinguished between PSP patients with versus patients without apathy and impulsivity.

Conclusions: The co-existence of apathy and impulsivity in PSP suggests that these neuropsychiatric features may share similar risk factors and etio-pathogenetic mechanisms. Apathy and impulsivity should be jointly assessed when planning symptomatic treatments for detrimental behavioral problems caused by PSP.
\end{abstract}

Progressive supranuclear palsy (PSP) is a neurodegenerative disorder caused by neuronal and glial aggregation of hyperphosphorylated 4- $\mathrm{R}$ tau isoforms, as part of the spectrum of diseases caused by frontotemporal lobar degeneration. ${ }^{1}$ PSP is characterized by postural instability, akinetic rigidity, and oculomotor dysfunction. ${ }^{1}$ Patients with PSP often display prominent neuropsychiatric problems, including apathy and impulsivity. ${ }^{2-4}$ In PSP and related disorders, apathy and impulsivity reduce survival $^{5}$ and predict loss of functional independence. ${ }^{6}$

Apathy and impulsivity are common, multifactorial, and frequently co-existent symptoms. Apathy has affective, cognitive, and behavioral components, ${ }^{7,8}$ with a resultant loss of interest in activities and difficulty initiating actions. ${ }^{9}$ Impulsivity encompasses actions that are premature, without foresight of deleterious consequences or made as a result of a failure to inhibit contextually inappropriate responses. ${ }^{10,11}$ Apathy and impulsivity tend to occur together in PSP, ${ }^{4,12}$ frontotemporal dementia, ${ }^{11}$ and Parkinson's disease. $^{13}$

This co-existence of apathy and impulsivity argues against the motivational spectrum hypothesis, with apathy and impulsivity at opposite ends of the spectrum. ${ }^{14,15}$ There are several and not necessarily mutually exclusive explanations for the reported co-

${ }^{1}$ School of Clinical Medicine, Cambridge University Hospitals, Cambridge, United Kingdom; ${ }^{2}$ Department of Clinical Neurosciences, University of Cambridge,

Cambridge, United Kingdom; ${ }^{3}$ Cognition and Brain Sciences Unit, University of Cambridge, Cambridge, United Kingdom; ${ }^{4}$ Istituto di Bioimmagini e Fisiologia Molecolare, Consiglio Nazionale Ricerche, Milan, Italy

*Correspondence to: Dr Luca Passamonti, Department of Clinical Neurosciences, University of Cambridge, Cambridge CB2 0SZ, UK; E-mail: lp337@medschl. cam.ac.uk

Keywords: apathy, impulsivity, progressive supranuclear palsy.

This is an open access article under the terms of the Creative Commons Attribution License, which permits use, distribution and reproduction in any medium, provided the original work is properly cited.

Received 23 February 2021; revised 21 July 2021; accepted 21 August 2021.

Published online 16 September 2021 in Wiley Online Library (wileyonlinelibrary.com). DOI: 10.1002/mdc3.13339 
existence of apathy and impulsivity. First, it may be chance, given that both are common. Second, they may have a shared neuroanatomical basis, ${ }^{12,16}$ including the degeneration of analogous fronto-striato-thalamo-cortical circuits, which represent actions, rewards, and inhibitory control. ${ }^{11}$ Third, there may be shared neurochemical systems mediating apathy and impulsivity, ${ }^{11,17}$ which would be especially relevant for pharmacological treatments. In clinical practice, dopaminergic agents do not clearly ameliorate apathy or impulsivity, ${ }^{11}$ but serotonergic and noradrenergic strategies might be effective. ${ }^{18-20}$ Fourth, individuals may express apathy and impulsivity at different times, perhaps alternating between them. Detailed temporal studies are lacking, but if the few actions made by an apathetic individual were premature, high-risk and contextually disinhibited, this would be best interpreted as truly concurrent apathy and impulsivity.

Despite many studies of apathy and impulsivity in PSP, ${ }^{2,4,12,16,21-24}$ basic questions about their co-existence in PSP remain unanswered. For example, is their co-existence more frequent than by chance? To what extent do these behavioral problems relate to other contextually inappropriate behaviors, such as emotional lability (or "incontinence")? Emotional lability refers to sudden, rapid, exaggerated, and uncontrollable bursts of laughing or crying that are spontaneous and often inappropriate to the social context. During these episodes, patients may report congruent emotional sentiments (ie, feeling sad while crying) or an incongruity between the external emotional appearance and their "internal" feelings (ie, not feeling sad while crying). ${ }^{25}$ Emotional lability has been associated to lesions in the pontine nuclei, cerebellum, and frontal lobes, ${ }^{26}$ but a link to impulsivity, apathy or both has not been investigated.

This study has three aims. First, to test the hypothesis that apathy and impulsivity co-exist in PSP, at the level of individual patients. Second, to test the association between presence of emotional lability and apathy or impulsivity. Third, to compare demographic, clinical, and cognitive features across PSP patients with or without apathy and impulsivity.

\section{Methods}

\section{Participants}

This study included a sample of 154 patients with a clinical diagnosis of probable or possible PSP, identified by retrospective rediagnosis according to the 2017 Movement Disorder Society (MDS) criteria. ${ }^{1}$ The data were extracted from research records and electronic medical records at a tertiary referral centre, from the Pick's Disease and Progressive Supranuclear Palsy Prevalence and Incidence protocol (12/EE/0475), the "Diagnosis and Prognosis in PSP and CBD" protocol (07/q0102/3) and the Prospective Evaluation of Parkinson's Plus and Related Disorders Protocol (07/Q0102/3). Sixty-four patients had pathological confirmation of PSP, via the Cambridge Brain Bank. Two patients were excluded from the study as they received a pathological diagnosis that differed from their clinical diagnosis of PSP. Exclusion criteria were the following: diagnosis of other neurodegenerative conditions including corticobasal syndrome (although the PSP-CBS subtype as per the 2017 MDS criteria was included), primary akinesia of gait freezing (PAGF), Lewy body dementia, idiopathic Parkinson's disease, Alzheimer's disease, stroke, cancer, and normal pressure hydrocephalus.

Clinical and demographic features included gender, age at symptom onset, disease duration, and PSP subtype. ${ }^{1}$ Disease severity, motor symptoms, cognition, and behavioral problems were respectively assessed via the Progressive Supranuclear PalsyRating Scale (PSPRS), Unified Parkinson's Disease Rating Scale (UPDRS-part III), Addenbrooke's Cognitive ExaminationRevised (ACE-R), Frontal Assessment Battery (FAB), and Cambridge Behavioral Inventory-revised (CBI-R). Behavioral changes were also identified throughout qualitative analysis of clinical letters and notes, coded in binary terms (absent or present) based on descriptions of apathy, impulsivity, and emotional lability, as reported in Appendix S1. In order for these behavioral changes to be encoded, patients were required to have three or more qualitative features of apathy, impulsivity or emotional lability, and these features had to be reported consistently across three or more clinical follow-ups. The assessment of behavioral changes was supplemented by scores in the CBI-R (questions on Motivation and Abnormal behavior) and PSPRS (questions on withdrawal and irritability, see Appendix S3 for details). Clinical letters were available in all patients. PSPRS was available in $n=79$ patients, CBI in $n=52$ patients. Twenty-seven patients completed both CBI and PSPRS. For these patients, apathy and impulsivity were coded as present or absent if the binary coding was fully concordant across the two scales. For patients with either CBI or PSPRS, the binary coding of behavioral abnormalities from the clinical letters had a $100 \%$ concordance rate to the items endorsing apathy and impulsivity in the CBI and PSPRS. This is illustrated in Appendix S5. The distribution of CBI scores endorsing apathy and impulsivity is illustrated in Appendix S4.

The Progressive Supranuclear Palsy-Rating Scale (PSP-RS) is a 28 -item clinical scale measuring the overall disease severity. It assesses clinical impairment across functional, motor and behavioral domains, and scores patients from 0 to 100 . The UPDRS is a clinical scale used to evaluate the severity of both motor and non-motor aspects of Parkinson's disease. In this study, UPDRS motor scores are used to quantify the severity of PSP patients' motor symptoms. The motor section of the UPDRS scale scores patients from 0 to 52, across 13 subsections. The Addenbrooke's Cognitive Examination-Revised (ACE-R) assesses the degree of cognitive impairment by quantifying patient performance in the following domains - attention/orientation, memory, verbal fluency, language, and visuo-spatial functions. This brings the maximum possible ACE-R score to 100. The Frontal Assessment Battery (FAB) quantifies the degree of frontal lobe dysfunction by assessing various aspects of executive functions. FAB scores range from 0 to 18 , with higher scores indicating better executive functions. On the other hand, the Cambridge Behavioral Inventory (CBI) is a 45-item informant-based questionnaire designed to assess neuropsychiatric symptoms in 


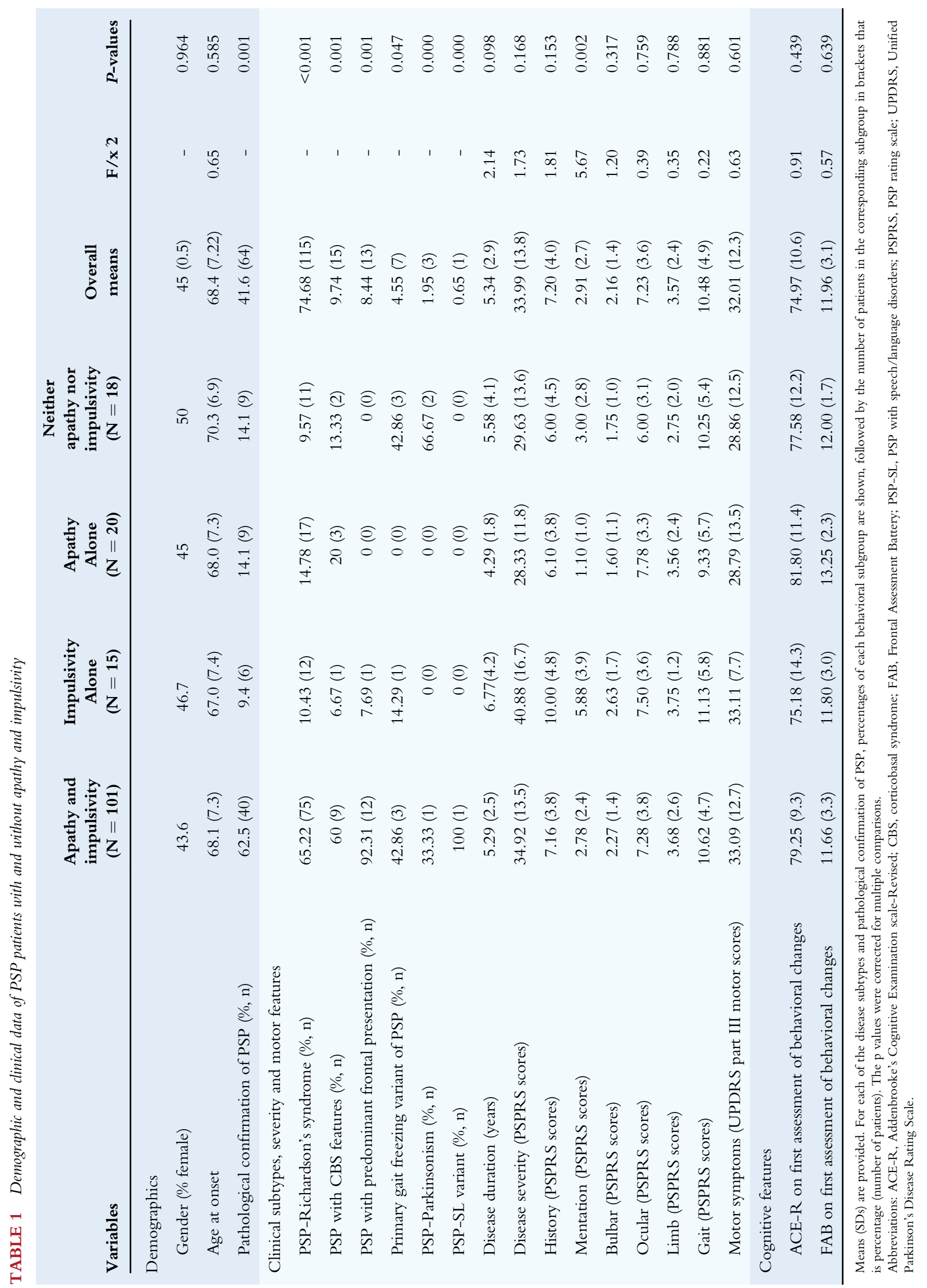




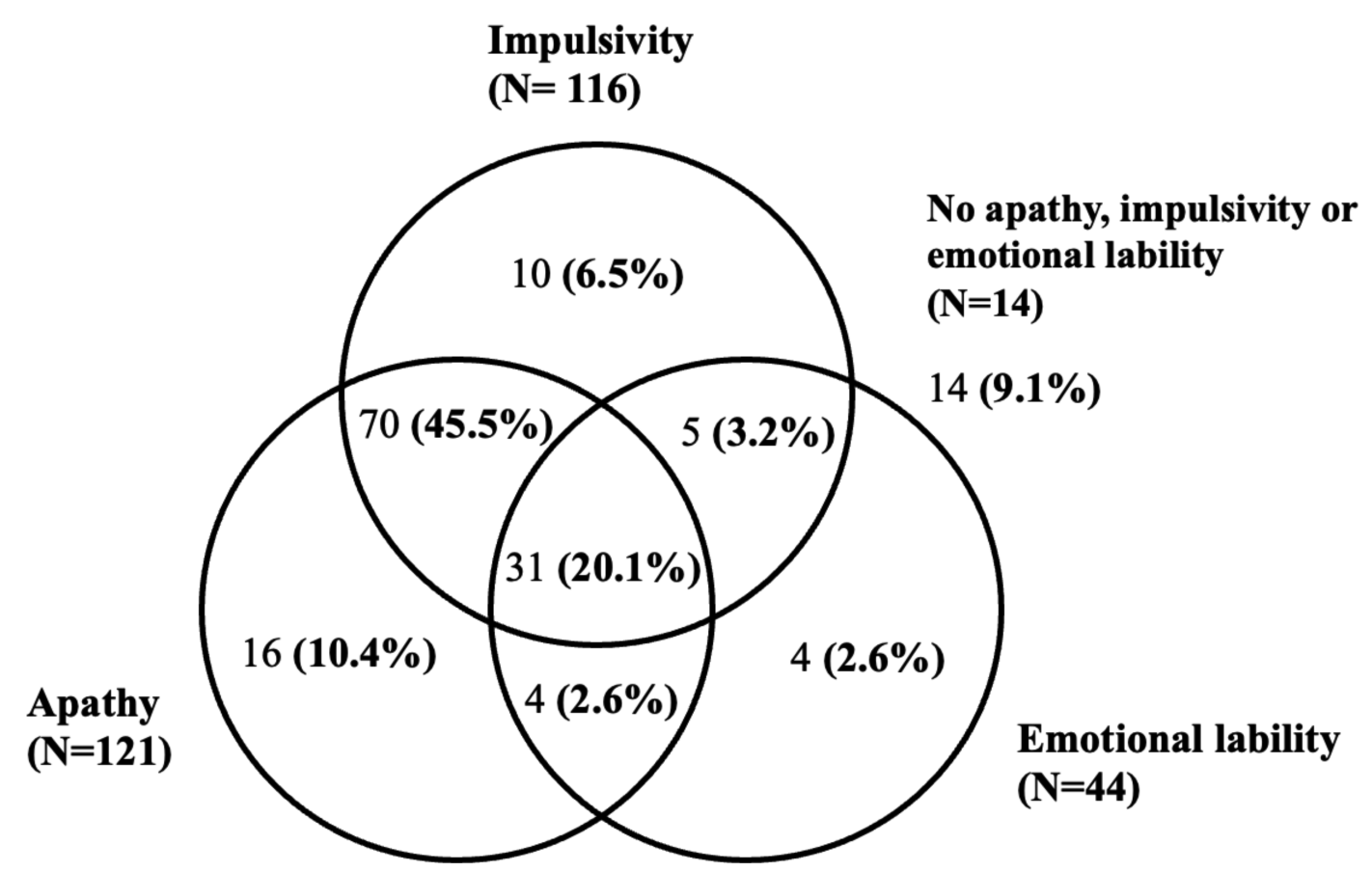

FIG. 1. Distribution of patients with apathy, impulsivity and emotional lability (number of patients given, followed by percentage of total patient sample in parentheses).

neurodegenerative disorders. It quantifies the severity of behavioral symptoms based on their frequency. CBI scores range from 0 to 180 (with higher scores reflecting a higher level of neuropsychiatric impairment).

We used the ACE-R and FAB scores recorded within 3 months of the first clinical record of apathetic or impulsive behavioral changes. PSP patients without this information recorded within 3 months of their first behavioral presentation were excluded from the analysis of ACE-R and FAB scores $(\mathrm{n}=35)$. For patients who experienced neither behavioral changes, we employed the scores measured within 3 months of their diagnosis of PSP. People who were unable to complete the assessments, or those lacking ACE-R/PSPRS/FAB measures were also excluded from the analysis of the specific clinical scores they lacked ( $\mathrm{n}=16$ patients). Despite being excluded from the analyses that needed the ACE-R, PSPR or FAB measures, these $\mathrm{n}=51$ patients were still included in the overall study, including for example the comparison of behavioral changes, demographic variables and other clinical measures.

\section{Statistical Analyses}

Statistical analyses used IBM SPSS (version 27.0) for frequentist analyses and JASP (Version 0.14) for Bayesian analyses. Chisquared tests for the co-occurrence of apathy, impulsivity, and emotional lability were conducted. One-way analyses of variance (ANOVAs) were used to compare age at onset, disease duration,
ACE-R, FAB, and PSPRS scores (at the first presentation) between groups with both apathy and impulsivity, only apathy or impulsivity or neither of them. Bayesian ANOVAs were used to test the relative evidence of null (no difference) and alternate (difference) hypotheses.

\section{Results}

Patients comprised six subgroups, as shown in Table 1. Most participants (74\%) had PSP-Richardson's syndrome. The remainders spanned PSP with CBS features (10\%), PSP with frontal presentation (8\%), PSP with primary gait freezing (4\%), PSPparkinsonism $(2 \%)$, or PSP with speech and language presentation (1\%).

The prevalence of apathy, impulsivity, and emotional lability is shown in Fig. 1. Overall, $75 \%$ of the patient group $(n=116)$ had impulsive behavioral changes; $79 \%(\mathrm{n}=121)$ had apathetic behavioral problems; and $29 \%$ of patients $(n=44)$ manifested emotional lability. Twenty percent displayed all three behavioral changes. Two-thirds had both apathetic and impulsive behavior reported.

Table 2 shows the relationship between apathy and impulsivity. There was a positive association between them $\left(\chi_{(1154)}^{2}=18.2\right.$, $P<0.001$ continuity corrected)). In other words, the probability of being apathetic increases with impulsivity.

Table 2 also shows the relationship between emotional lability and impulsivity. There was no significant relationship 
TABLE 2 Contingency tables for apathy, impulsivity, and emotional lability in PSP patients

Contingency table for apathy and impulsivity. $\chi_{(1154)}^{2}=18.2, P<0.001$ (continuity corrected)

\begin{tabular}{|c|c|c|c|c|}
\hline & \multicolumn{3}{|c|}{ Impulsivity (n) } & \multirow[b]{2}{*}{ Total (n) } \\
\hline & & & Yes & \\
\hline \multirow[t]{2}{*}{ Apathy (n) } & No & 18 & 15 & 33 \\
\hline & Yes & 20 & 101 & 121 \\
\hline Total & \multicolumn{2}{|c|}{38} & 116 & 154 \\
\hline
\end{tabular}

Contingency table for emotional lability and impulsivity. $\chi_{(1154)}^{2}<1$, ns (continuity corrected)

\begin{tabular}{lccccc}
\hline & \multicolumn{2}{c}{ Emotional lability (n) } & Yes & Total (n) \\
\cline { 2 - 5 } & & No & 30 & 8 & 38 \\
Impulsivity (n) & No & & 80 & 36 & 116 \\
& Yes & & 44 & 154 \\
\hline
\end{tabular}

Contingency table for emotional lability and apathy. $\chi_{(1154)}^{2}<1$ (corrected)

\begin{tabular}{|c|c|c|c|c|}
\hline & \multicolumn{3}{|c|}{ Emotional lability (n) } & \multirow[b]{2}{*}{ Total (n) } \\
\hline & & & Yes & \\
\hline \multirow[t]{2}{*}{ Apathy (n) } & No & 24 & 9 & 33 \\
\hline & Yes & 86 & 35 & 121 \\
\hline Total & \multicolumn{2}{|c|}{110} & 44 & 154 \\
\hline \multicolumn{5}{|c|}{ Contingency table for co-existent apathy, impulsivity, and emotional lability. $\chi^{2}{ }_{(1154)}<1$, ns (corrected). } \\
\hline & \multicolumn{3}{|c|}{ Emotional lability (n) } & \\
\hline & \multicolumn{2}{|c|}{ No } & Yes & Total (n) \\
\hline \multirow{2}{*}{$\begin{array}{l}\text { Co-existent apathy and } \\
\text { impulsivity (n) }\end{array}$} & No & 41 & 13 & 54 \\
\hline & Yes & 69 & 31 & 100 \\
\hline Total (n) & \multicolumn{2}{|c|}{110} & 44 & 154 \\
\hline
\end{tabular}

$\left(\chi_{(1154)}^{2}<1, n s\right)$. In other words, the probability of being impulsive was independent of emotional lability. Table 2 illustrates the relationship between emotional lability and apathy. There was no significant relationship $\left(\chi_{(1154)}^{2}<1, n s\right)$. In other words, the probability of being apathetic was independent of emotional lability. Finally, Table 2 shows the relationship between emotional lability and co-existent apathy and impulsivity. There was no significant relationship $\left(\chi_{(1154)}^{2}<1, n s\right)$.

There were sixty-four patients who had pathological confirmation of PSP. The prevalence of apathy, impulsivity, and emotional lability in the patient cohort with pathological confirmation of PSP is shown in Fig. 2. Overall, $72 \%$ of those with pathological confirmation of PSP ( $\mathrm{n}=46$ ) had impulsive behavioral changes; $77 \%$ $(\mathrm{n}=49)$ had apathetic behavioral problems; and 30\% of patients ( $\mathrm{n}=19)$ manifested emotional lability. 19\% displayed all three behavioral changes, and $62.6 \%$ had both apathetic and impulsive behavior reported. Table 3 illustrates a positive association between apathy and impulsivity in PSP patients with pathological confirmation of diagnosis $\left.\chi_{(1154)}^{2}=7.89, P<0.005\right)$. Table 3 also shows that there is no significant relationship between emotional lability and impulsivity in these patients $\chi_{(1154)}^{2}<1$, ns) and that there is no significant relationship between emotional lability and apathy in this patient group $\left.\chi_{(1154)}^{2}<1, n s\right)$. These results confirm our findings in the main cohort. This is further illustrated in Appendix S7.

Comparing groups with apathy, impulsivity, neither or both with the frequentist tests (Table 1), we found no significant difference by sex, age at onset, disease duration, disease severity (as indexed by the PSPRS), global cognitive screening tests (ACE-R, FAB). Bayesian analyses confirmed these null findings with positive or very strong evidence in favor of the null hypothesis $\left(\mathrm{BF}_{10}<1 / 3\right.$ or $<1 / 10$ respectively; Supplementary Materials). We also found no significant between-groups differences in the PSPRS sub-scores, except for mentation. Upon further analyses of the data, we found that higher mentation PSPRS sub-scores related to a higher likelihood of displaying impulsivity, but not apathy $(P<0.05)$. 


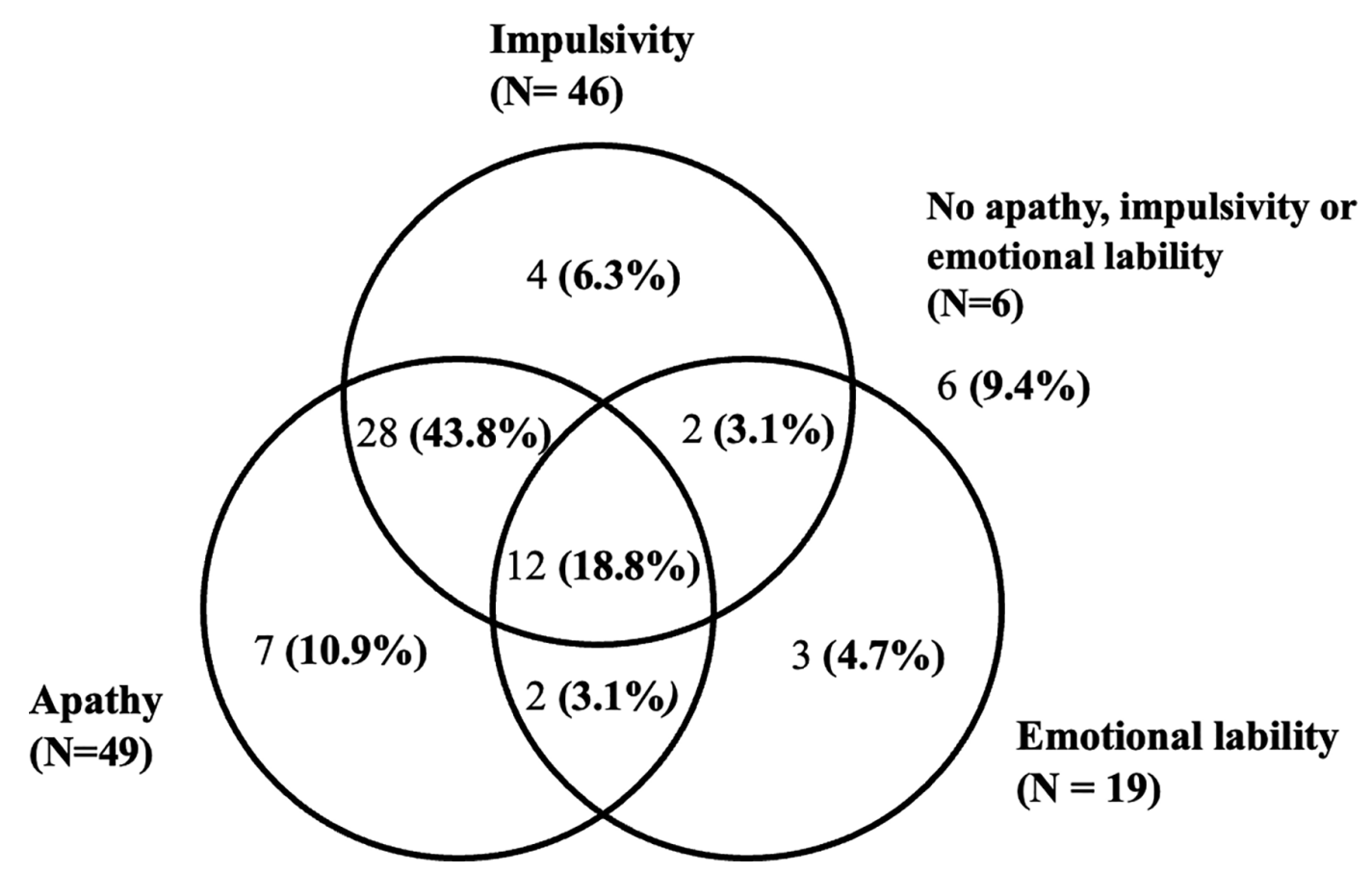

FIG. 2. Distribution of behavioral abnormalities in patients with pathological confirmation of PSP (number of patients given, followed by percentage of total patient sample).

\section{Discussion}

This study confirms the high frequency of reported co-existence of apathy and impulsivity in PSP, with a positive relationship between them. Neither apathy nor impulsivity were associated with emotional lability. Approximately three-quarters of people with PSP had apathy, in keeping with previous reports that apathy is the most common neuropsychiatric feature in PSP. ${ }^{2-4}$ Most patients with apathy also manifested impulsive behaviors, more than by chance. The prevalence of impulsivity in our cohort was higher than previously reported (74\% versus $32 \%$ to $43 \%{ }^{3,4,23}$ ). This disparity may be due to methodological differences to assess impulsivity across studies: patient self-ratings, clinician judgment of behavioral changes, or carer reports using tools such as the Cambridge Behavioral Inventory (CBI), Neuropsychiatric Inventory (NPI) or Frontal Behavioral Inventory (FBI).

The strong concomitance of apathy and impulsivity accords with previous studies of other syndromes associated with frontotemporal lobar degeneration ${ }^{9,2,22,27}$ and Parkinson's disease. ${ }^{9}$ Such a positive relationship across multiple disorders suggests that apathy and impulsivity may share similar risk factors and etiopathogenetic mechanisms. ${ }^{22,28,29}$ For example, apathy and impulsivity have common correlates in the white-matter tracts connecting the prefrontal cortex, basal ganglia, temporal poles, and brainstem ${ }^{22}$ as well as gray-matter atrophy across the frontal cortex. ${ }^{12,28}$ Despite molecular pathological differences, there is convergence onto similar neural circuits across different neurodegenerative disorders. This signals the need to study apathy and impulsivity together rather than in isolation, ${ }^{11,12}$ and highlights the potential for joint therapeutic strategies, rather than dopaminergic antagonism between apathy (or akinesia) and impulsivity. ${ }^{30}$ In view of their positive association, apathy and impulsivity cannot be simply conceptualized as opposite extremes of a dopamine-dependent spectrum, with apathy arising from a hypodopaminergic state and impulsivity from a hyperdopaminergic state. $^{14,15}$

Other neurotransmitters are likely to influence apathy and impulsivity. For example, serotonin is reduced in several neurodegenerative disorders, and serotonergic manipulations through serotonin reuptake inhibition can ameliorate deficits in response inhibition in patients with Parkinson's disease and frontotemporal dementia. ${ }^{18,20}$ Apathy and impulsivity may also be attributed to noradrenergic deficits. ${ }^{31}$ For example, noradrenergic reuptake inhibition improves response inhibition and restores the function of inhibitory control networks in Parkinson's disease. ${ }^{19,32}$

Emotional lability was not positively associated with either apathy or impulsivity. This suggests that emotional lability arises from dysfunctions in separate neural systems, even though it was relatively common in PSP (with a prevalence of 20\%). For example, whilst fronto-striatal systems are affected in apathy and impulsivity, ${ }^{11}$ fronto-ponto-cerebellar circuits have been linked to emotional lability. ${ }^{33}$ Both of these neuroanatomical systems show diffuse tau pathology and neurodegeneration in PSP.

With the exception of the mentation component of the PSPRS subscale, there were no specific demographic or clinical characteristics linked to apathy and impulsivity in PSP. This is in contrast with 
TABLE 3 Contingency tables for apathy, impulsivity, and emotional lability in PSP patients with pathological confirmation of diagnosis

Contingency table for apathy and impulsivity. $\chi_{(1154)}^{2}=7.89, P<0.005$ (continuity corrected)

\begin{tabular}{lcccccc}
\hline & & Impulsivity (n) & & Yes & Total (n) \\
\cline { 2 - 5 } & & No & 9 & 6 & 15 \\
Apathy (n) & No & 9 & 40 & 49 \\
Total & Yes & & 46 & 64 \\
\hline
\end{tabular}

Contingency table for emotional lability and impulsivity. $\chi_{(1154)}^{2}<1$, ns (continuity corrected)

\begin{tabular}{lccccc}
\hline & \multicolumn{2}{c}{ Emotional lability (n) } & & & Total (n) \\
\cline { 2 - 5 } & & No & 13 & 5 & 18 \\
Impulsivity (n) & No & 32 & 14 & 46 \\
Total (n) & Yes & & 45 & 19 & 64 \\
\hline
\end{tabular}

Contingency table for emotional lability and apathy. $\chi_{(1154)}^{2}<1$ (corrected)

\begin{tabular}{|c|c|c|c|c|}
\hline & \multicolumn{3}{|c|}{ Emotional lability (n) } & \multirow[b]{2}{*}{ Total (n) } \\
\hline & & & Yes & \\
\hline \multirow[t]{2}{*}{ Apathy (n) } & No & 10 & 5 & 15 \\
\hline & Yes & 35 & 14 & 49 \\
\hline Total & \multicolumn{2}{|c|}{45} & 19 & 64 \\
\hline \multicolumn{5}{|c|}{ Contingency table for co-existent apathy, impulsivity, and emotional lability. $\chi^{2}{ }_{(1154)}<1$, ns (corrected). } \\
\hline & \multicolumn{3}{|c|}{ Emotional lability (n) } & \\
\hline & \multicolumn{2}{|c|}{ No } & Yes & Total (n) \\
\hline \multirow{2}{*}{$\begin{array}{l}\text { Co-existent apathy } \\
\text { and impulsivity (n) }\end{array}$} & No & 17 & 7 & 24 \\
\hline & Yes & 28 & 12 & 40 \\
\hline Total (n) & \multicolumn{2}{|c|}{45} & 19 & 64 \\
\hline
\end{tabular}

some studies showing an association between apathy and executive functions. ${ }^{4,34}$ However, another single study did not report such association. ${ }^{23}$ It was also surprising that the Frontal Assessment Battery did not distinguish between patients with and without behavioral changes. A possibility is that the power of our study was insufficient to detect this effect, we therefore do not rule out a potential correlation between executive function and apathy or impulsivity. Some of the mental symptoms described in the PSPRS, such as disorientation, bradyphrenia and utilizing behaviors related to impulsivity in PSP and may be are mediated by overlapping brain networks. Although it is difficult to directly compare studies using different methodologies and assessment tools, the discrepancies may also depend on specific subcomponents of apathy and impulsivity, ${ }^{11,12}$ which we did not differentiate in this study. ${ }^{3,4,34}$ Furthermore, while apathy, impulsivity and executive functions may be mediated by shared fronto-striatal circuits, progressive dysfunction of the circuits involved may not occur in parallel in PSP.
Our work has limitations. It is a retrospective analysis, albeit drawing on patients with PSP in longitudinal observational studies. We relied on clinical diagnosis, although $n=64$ patients had pathological confirmation of PSP diagnosis, and clinicopathological confirmation is typically very high in PSP. Indeed, in this cohort, only two patients received a pathological diagnosis that differed from their clinical diagnosis of PSP. We also included reports of behavioral features over multiple clinical follow-ups, as it may be that apathy and impulsivity are intermittent rather than constant features. Higher temporal resolution of assessments would be needed to confirm this. Such assessments may rely on carer reports, given a lack of insight into behavioral changes, which can be present in PSP.

We recognize that the qualitative assessment of apathy and impulsivity is an important limitation of our study. Nevertheless, the qualitative coding of apathy and impulsivity was well concordant with the coding of behavioral changes from the PSPRS and CBI scores (see Tables in Appendix S5). In addition, qualitative and quantitative scales have their own strengths and weaknesses. 
Quantitative scales such as CBI are more generalisable across studies relative to qualitative data such as clinical letters, and provide more details on the severity of behavioral abnormalities. On the other hand, clinical letters contain assessment from multiple informants, including patients, carers, and doctors. For this reason, clinical letters are less vulnerable to variability relating to single informants, or other factors such as carer distress. In our tertiary clinic for PSP, clinical letters emphasize patients' unique perspective, and provide a holistic overview of the various aspects of apathy and impulsivity. They also report real-life examples of apathy and impulsivity. A potential caveat of using clinical letters is the confirmation bias involved in ascertaining if patients had certain behavioral features, such as apathy and impulsivity. However, we strived to maintain clear selection criteria when assessing these neuropsychiatric problems, drawing on multiple informants and measures. These criteria used the thresholds set out in Appendixes S1-S3. Patients were required to have $\geq 3$ features of apathy, impulsivity or emotional lability, and these features had to be reported persistently across $\geq 3$ clinical follow-ups.

Another limitation of our study is the dichotomic assessment of apathy and impulsivity, rather than the use of continuous ratings. This was pragmatic and enabled data to be drawn from a larger cohort, and over a longer period of time. Our crosssectional findings need to be replicated across other centres and in longitudinal studies and would benefit from systematic measures with a dynamic range suitable for PSP patients. Given the crosssectional nature of our findings, it is plausible that we may have underestimated the prevalence of these neuropsychiatric features. However, apathy and impulsivity are often present early in PSP, ${ }^{28,35}$ and our average follow-up was 5.3 years from symptom onset.

To conclude, our study highlights the co-morbid nature of apathy and impulsivity in PSP, and their independence from emotional lability. This informs future studies of the neural correlates of apathy and impulsivity, their risk factors, and new therapeutic strategies to reduce apathy and impulsivity.

\section{Author Roles}

(1) Research project: A. Conception, B. Organization, C. Execution; (2) Statistical Analysis: A. Design, B. Execution, C. Review and Critique; (3) Manuscript: A. Writing of the first draft, B. Review and Critique.

$$
\begin{aligned}
& \text { ZQK.: 1B, 1C, 2A, 2B, 3A, 3B } \\
& \text { AGM.: 2C, 3B } \\
& \text { TR.: 2C, 3B } \\
& \text { JR.: 1A, 1B, 2C, 3B } \\
& \text { LP.: 1A, 1B, 2A, 2C, 3B }
\end{aligned}
$$

\section{Disclosures}

Ethical Compliance Statement: Written informed consent was obtained from all participants. Ethical approval was given by the East of England - Cambridge Central Research Ethics Committee for the Pick's Disease and Progressive Supranuclear Palsy
Prevalence and Incidence protocol (12/EE/0475) (October 2015) and by the East of England-Essex Research Ethics Committee for the Diagnosis and prognosis in Progressive Supranuclear Palsy and Corticobasal Degeneration protocol (07/Q0102/3) (March 2007). All necessary patient/participant consent has been obtained and the appropriate institutional forms have been archived. We confirm that we have read the Journal's position on issues involved in ethical publication and affirm that this work is consistent with those guidelines.

Funding Sources and Conflicts of Interest: This study was co-funded by the Cambridge University Centre for ParkinsonPlus (RG95450) and the Medical Research Council (RG91365). The Cambridge Brain Bank is supported by the NIHR Cambridge Biomedical Research Centre. The authors also declare that there are no conflicts of interest relevant to this work.

Financial Disclosures for the previous 12 months: James B. Rowe serves as an associate editor to Brain and is a nonremunerated trustee of the Guarantors of Brain, Darwin College and the PSP Association (UK). He provides consultancy to Asceneuron, Biogen, UCB and has research grants from AZMedimmune, Janssen, Lilly as industry partners in the Dementias Platform UK. The authors declare that there are no additional disclosures to report.

\section{References}

1. Höglinger GU, Respondek G, Stamelou M, et al. Clinical diagnosis of progressive supranuclear palsy: the movement disorder society criteria. Mov Disord 2017;32:853-864.

2. Aarsland D, Litvan I, Larsen JP. Neuropsychiatric symptoms of patients with progressive supranuclear palsy and Parkinson's disease. J Neuropsychiatry Clin Neurosci 2001;13:42-49.

3. Cordato NJ, Halliday GM, Caine D, Morris JGL. Comparison of motor, cognitive, and behavioral features in progressive supranuclear palsy and Parkinson's disease. Mov Disord 2006;21:632-638.

4. Litvan I, Mega MS, Cummings JL, Fairbanks L. Neuropsychiatric aspects of progressive supranuclear palsy. Neurology 1996;47:1184-1189.

5. Lansdall CJ, Coyle-Gilchrist ITS, Vázquez Rodríguez P, et al. Prognostic importance of apathy in syndromes associated with frontotemporal lobar degeneration. Neurology 2019;92:e1547-e1557.

6. Murley AG, Rouse MA, Coyle-Gilchrist ITS, et al. Predicting loss of independence and mortality in frontotemporal lobar degeneration syndromes. J Neurol Neurosurg Psychiatry 2021(92):737-744. https://doi.org/ 10.1136/jnnp-2020-324903.

7. Ducharme S, Price BH, Dickerson BC. Apathy: a neurocircuitry model based on frontotemporal dementia. J Neurol Neurosurg Psychiatry 2018;89:389-396.

8. Johnson E, Kumfor F. Overcoming apathy in frontotemporal dementia: challenges and future directions. Curr Opin Behav Sci 2018;22:82-89.

9. Scott BM, Eisinger RS, Burns MR, Lopes J, Okun MS, Gunduz A, Bowers D. Co-occurrence of apathy and impulse control disorders in Parkinson disease. Neurology 2020;95:e2769-e2780.

10. Dalley JW, Robbins TW. Fractionating impulsivity: neuropsychiatric implications. Nat Rev Neurosci 2017;18:158-171.

11. Passamonti L, Lansdall C, Rowe J. The neuroanatomical and neurochemical basis of apathy and impulsivity in frontotemporal lobar degeneration. Curr Opin Behav Sci 2018;22:14-20.

12. Lansdall CJ, Coyle-Gilchrist ITS, Jones PS, et al. Apathy and impulsivity in frontotemporal lobar degeneration syndromes. Brain 2017;140:1792-1807.

13. Drew DS, Muhammed K, Baig F, et al. Dopamine and reward hypersensitivity in Parkinson's disease with impulse control disorder. Brain 2020; 143:2502-2518.

14. Sierra M, Carnicella S, Strafella AP, et al. Apathy and impulse control disorders: Yin \& Yang of dopamine dependent behaviors. J Parkinsons Dis 2015;5:625-636. 
15. Sinha N, Manohar S, Husain M. Impulsivity and apathy in Parkinson's disease. J Neuropsychol 2013;7:255-283.

16. Murley AG, Rouse MA, Coyle-Gilchrist ITS, et al. Redefining the multidimensional clinical phenotypes of frontotemporal lobar degeneration syndromes. Brain 2020;143:1555-1571.

17. Murley AG, Rowe JB. Neurotransmitter deficits from frontotemporal lobar degeneration. Brain 2018;141:1263-1285.

18. Hughes LE, Rittman T, Regenthal R, Robbins TW, Rowe JB. Improving response inhibition systems in frontotemporal dementia with citalopram. Brain 2015;138:1961-1975.

19. Ye Z, Altena E, Nombela C, et al. Improving response inhibition in Parkinson's disease with Atomoxetine. Biol Psychiatry 2015;77: 740-748.

20. Ye Z, Altena E, Nombela C, et al. Selective serotonin reuptake inhibition modulates response inhibition in Parkinson's disease. Brain 2014; 137:1145-1155.

21. Gerstenecker A. The neuropsychology (broadly conceived) of multiple system atrophy, progressive Supranuclear palsy, and Corticobasal degeneration. Arch Clin Neuropsychol 2017;32:861-875.

22. Lansdall CJ, Coyle-Gilchrist ITS, Jones PS, et al. White matter change with apathy and impulsivity in frontotemporal lobar degeneration syndromes. Neurology 2018;90:e1066-e1076.

23. Gerstenecker A, Duff K, Mast B, Litvan I. ENGENE-PSP study group. Behavioral abnormalities in progressive supranuclear palsy. Psychiatry Res 2013;210:1205-1210.

24. Zhang J, Rittman T, Nombela C, et al. Different decision deficits impair response inhibition in progressive supranuclear palsy and Parkinson's disease. Brain 2016;139:161-173.

25. Chiu HF. Psychiatric aspects of progressive supranuclear palsy. Gen Hosp Psychiatry 1995;17:135-143.

26. Damasio AR, Grabowski TJ, Bechara A, et al. Subcortical and cortical brain activity during the feeling of self-generated emotions. Nat Neurosci 2000;3:1049-1056.

27. Kertesz A, Martinez-Lage P, Davidson W, Munoz DG. The corticobasal degeneration syndrome overlaps progressive aphasia and frontotemporal dementia. Neurology 2000;55:1368-1375.

28. Vecchio I, Tornali C, Malaguarnera G, Bragazzi NL, Malaguarnera M. Progressive supranuclear palsy: neuropsychopathological, therapeutical and bioethical aspects. Curr Alzheimer Res 2018;15:959-963.

29. Husain M, Roiser JP. Neuroscience of apathy and anhedonia: a transdiagnostic approach. Nat Rev Neurosci 2018;19:470-484.

30. Napier TC, Corvol JC, Grace AA, et al. Linking neuroscience with modern concepts of impulse control disorders in Parkinson's disease. Mov Disord 2015;30:141-149.
31. Loued-Khenissi L, Preuschoff K. Apathy and noradrenaline: silent partners to mild cognitive impairment in Parkinson's disease? Curr Opin Neurol 2015;28:344-350.

32. Borchert RJ, Rittman T, Passamonti L, et al. Atomoxetine enhances connectivity of prefrontal networks in Parkinson's disease. Neuropsychopharmacology 2016;41:2171-2177.

33. Parvizi J, Anderson SW, Martin CO, Damasio H, Damasio AR. Pathological laughter and crying: a link to the cerebellum. Brain 2001;124 1708-1719.

34. Santangelo G, Cuoco S, Pellecchia MT, et al. Comparative cognitive and neuropsychiatric profiles between Parkinson's disease, multiple system atrophy and progressive supranuclear palsy. J Neurol 2018;265:26022613.

35. Kobylecki C, Jones M, Thompson JC, et al. Cognitive-behavioural features of progressive supranuclear palsy syndrome overlap with frontotemporal dementia. J Neurol 2015;262:916-922.

\section{Supporting Information}

Supporting information may be found in the online version of this article.

Appendix S1. Qualitative selection criteria for apathy, impulsivity and emotional lability (linguistic analysis of clinical letters).

Appendix S2. Cambridge Behavioral Inventory score thresholds for apathy and impulsivity.

Appendix S3. PSP-RS (Progressive Supranuclear PalsyRating Scale) score thresholds for apathy and impulsivity.

Appendix S4. Distribution of CBI subscores representing apathy and impulsivity.

Appendix S5. Concordance between encoding of behavioral changes from clinical letters, CBI and PSP-RS clinical scores.

Appendix S6. Average CBI subscores of patients with and without apathy and impulsivity.

Appendix S7. Pathological confirmation of PSP in behavioral subgroups.

Appendix S8. Bayesian ANOVAs were used to examine differences between clinical variables across the behavioral subgroups. 